\title{
Green synthesis and characterization of palladium nanoparticles and its conjugates from solanum trilobatum leaf extract
}

\section{Amarnath Kanchana ${ }^{1, *}$, Saveetha Devarajan ${ }^{2}$ and Senniyanallur Rathakrishnan Ayyappan ${ }^{3}$}

An important area of research in nanotechnology deals with the synthesis of nanoparticles of different chemical compositions, sizes and controlled monodispersity. Currently, there is a growing need to develop environmentally benign nanoparticle synthesis in which no toxic chemicals are used in the synthesis protocol. Palladium nanoparticles (PdNp) are of interest because of their catalytic properties and affinity for hydrogen. Our protocol for the phyto-synthesis of PdNp under moderate pH and room temperature offers a new means to develop environmentally benign nanoparticles. Solanum trilobatum is enlightened in our present study as it is enriched with phytochemicals to reduce palladium chloride ions. Poly MVA a dietary supplement based on the nontoxic chemotherapeutic lipoic acid-palladium complex (LA-Pd) is been hypothesized as the new paradigm of cancer therapy. Hence forth we successfully conjugated lipoic acid (S-PdNp-LA) and vitamins (S-PdNp-Vitamin-LA) to palladium nanoparticles synthesised from Solanum trilobatum leaf extract. These nanoparticles (S-PdNp, S-PdNp-LA, S-PdNp-Vitamin-LA) were characterized with UV-Vis Spectroscopy, SEM and FTIR analysis, which revealed that S-PdNp are polydisperse and of different morphologies ranging from 60 70 $\mathrm{nm}$ (S-PdNp), 65 80 $\mathrm{nm}$ (S-PdNp-LA) and 75 100 $\mathrm{nm}$ (S-PdNp-Vitamin-LA) in size.

Keywords: Nanoparticles; Palladium; Lipoic acid; Palladium lipoic acid complex; Poly-MVA; Green synthesis; Solanum trilobatum

Citation: Amarnath Kanchana, Saveetha Devarajan and Senniyanallur Rathakrishnan Ayyappan "Green synthesis and characterization of palladium nanoparticles and its conjugates from solanum trilobatum leaf extract", Nano-Micro Lett. 2, 169-176 (2010). doi: 10.5101/nml.v2i3.p169-176

In modern nanoscience and nanotechnology the production of nanomaterials with the preferred quality is one of the most stimulating aspects. In addition, nanoparticles refer to nanoscale materials that have an aerodynamic equivalent diameter of 100 $\mathrm{nm}$ or less [1] yet, it has been shown that there is a strong tendency with several nanoscale materials sold commercially of agglomerating and therefore existing as much larger micrometer scale particles further complicating studies regarding exposure and effect. An important area of research in nanotechnology deals with the synthesis of metallic nanoparticles of different chemical compositions, sizes and controlled monodispersity [2]. To date, metallic nanoparticles are mostly prepared from nobel metals (ie. Ag, Pt, Au and Pd) [3]. Recently, the advances in fabrication of palladium nanoparticles (PdNPs) have gained great importance owing to their application both in heterogeneous and homogeneous catalysis, their high surfaceto-volume ratio and their high surface energy [4].

Biosynthetic methods employing either biological microorganisms or plant extracts have been suggested as possible ecofriendly alternatives to chemical and physical 
methods [5-19]. The rate of reduction of metal ions in the method of using plants has been found to be much faster than the method of using micro-organisms and stable. The synthesis of nanoparticles can be carried out by both intracellular and extracellular methods such as leaf broth, sundried leaves, fruits, growing plants on metal-rich soil, gold-rich agar media, etc. and the results obtained were positive in all cases. Extracellular nanoparticle synthesis using plant leaf extracts rather than whole plants would be more economical owing to easier downstream processing.

Biological processes using microorganisms, plants, and plant extracts have been used to synthesize nanoparticles of gold and silver with wide applications in the field of medicine, cancer treatment, drug delivery, commercial appliances, sensors, etc. There is relatively little knowledge in literatures concerning the biological synthesis of palladium nanoparticles. The only success was the recent findings on the production of PdNPs using coffee and tea extract [20]. It has also been found that the antioxidants such as geniposide, chlorogenic acid, crocins and crocetin were the reducing and stabilizing agents for synthesizing palladium nanoparticles ( 3 to $5 \mathrm{~nm}$ ) in water crude extract of Gardenia jasminoides Ellis' [21]. This study appeared to be a new promising biosynthetic nanocatalyst for the development of an industrial process. An extremely simple green approach that generated by Mallikarjuna et al., 2008 [22] in bulk quantities of nanocrystals $(20 \sim 60 \mathrm{~nm})$ of noble metals such as silver ( $\mathrm{Ag}$ ) and palladium (Pd) using coffee and tea extract at room temperature is noteworthy. Currently, however, the exact mechanism for the synthesis of palladium nanoparticles is unclear. Our protocol for the phyto-synthesis of palladium nanoparticles under moderate $\mathrm{pH}$ and room temperature offers a new means to develop environmentally benign nanoparticles.

Palladium Lipoic Acid (LAPd) is a formulation used in a prescription version called DNA Reductase and a dietary supplement called Poly MVA. The active ingredient is the palladium-lipoic acid polymer, which exists as a trimer of palladium-lipoic acid joined to thiamine. This arrangement is unique in that it allows the molecule to be both water and lipid soluble, as well as exist as a liquid crystal. This liquid crystalline structure allows it to store a great deal of energy and thus serve as a semiconductor [23-25]. "Palladium Lipoic Compounds" a specific class of compounds has become the centre piece of nontoxic cancer therapy in recent research.

It acts by modulating cellular energy helping the human body to build up the immune system and have an energetic life. Hence Poly-MVA that is a variant of Palladium Lipoic Acid
(LAPd) acts as an anti-oxidant supplement and helps us lead a life free from many diseases [26]. Poly-MVA is one of the first available formulations of Palladium Lipoic acid in the market which helps in fighting many diseases such as diabetic neuropathy, retinopathy which causes blindness, controls blood sugar levels in the body; prevent cardiomyopthay and helps in slowing the aging process by normalizing the free radicals in the body [27]. The palladium lipoic complexes in Poly-MVA work in novel ways that do not harm the body as a whole only the cancer cells specifically, partially by converting free radicals into a usable form of energy [28-29].

To the best of our knowledge, there are no reports on the reduction of aqueous palladium chlorate ions from the leaves of Solanum trilobatum. Hence in this study, we synthesized palladium nanoparticles using screened leaf extract of the Solanum trilobatum plant. Fourier-transform infrared spectroscopy (FTIR) analysis was used to identify the biomolecules responsible for reducing palladium ions and stabilizing the palladium nanoparticles formed. In addition this study is also focussed on the conjugation of palladium nanoparticles (S-PdNp) with Lipoic acid (LA-S-PdNp) and Vitamins (Vitamin-LA-S-PdNp).

\section{Experimental Section}

\section{Synthesis of palladium nanoparticles from Solanum trilobatum leaf extract}

The broth used for reduction of $\mathrm{PdCl}_{2}$ ions to palladium was prepared by taking $20 \mathrm{~g}$ of thoroughly washed and finely cut Solanum trilobatum leaves in a $500 \mathrm{ml}$ Erlenmeyer flask with $100 \mathrm{ml}$ sterile distilled water and then boiling the mixture for 1 min. The process of boiling the leaves leads to rupture of the walls of leaf cells and, thus, release of intra-cellular material into solution. After boiling, the solution was decanted, filtered and 5 $\mathrm{ml}$ of this broth was added to $100 \mathrm{ml}$ of $0.226 \mathrm{M} \mathrm{PdCl}_{2}$ aqueous solution.

\section{Palladium Nanoparticles from Solanum trilobatum leaf extract conjugated with Lipoic acid}

$15 \mathrm{~mL}$ of the leaf extract was taken and added $0.226 \mathrm{M}$ $\mathrm{PdCl}_{2}$ aqueous solution with $1 \mathrm{ml}$ of lipoic acid (15mg lipoic acid was dissolved in $0.5 \mathrm{ml}$ of ethanol and $0.5 \mathrm{ml}$ of water) and kept for incubation at room temperature for $24 \mathrm{hrs}$. The mixture was centrifuged at 4,500 rpm to separate the capped PdNPs. The pellet obtained was resuspended in $1 \mathrm{ml}$ of phosphate buffer ( $\mathrm{pH}$ 7). 
Palladium Lipoic Acid Complex Conjugated With Vitamins (Poly MVA)

The vitamin (Vitamins B1, B2, and B12) was added to the leaf extract containing palladium lipoic acid complex and kept for incubation at room temperature for 24 hours. Then after the incubation period the colour of the leaf extract was changed from yellow to brown colour. The mixture was centrifuged at 4,500 rpm to separate the capped PdNPs. The resulting palldium nanoparticle solution was purified by repeated centrifugation at $15,000 \mathrm{rpm}$ for $20 \mathrm{~min}$, with the pellet produced by this process redispersed in deionized water.

\section{UV-Vis spectroscopic Studies}

The bioreduction of $\mathrm{Pd}$ ions in aqueous solution was monitored by periodic sampling of aliquots $(0.2 \mathrm{ml})$ of the suspension, then diluting the samples with $2 \mathrm{ml}$ deionized water and subsequently measuring UV-vis spectra of the resulting diluents. UV-vis spectra of these aliquots were monitored as a function of time of reaction on a Hewlett-Packard diode array spectrophotometer (model HP-8452) operated at a resolution of $2 \mathrm{~nm}$.

\section{FTIR Observations}

Samples of the aqueous solution of the palladium nanoparticles were prepared by using centrifugation and suspended with phosphate buffer and analyses done by FTIR. Samples were measured by Bruker Tensor 27 FTIR spectrometer in attenuated total reflection mode (Pike Technologies, Gladi ATR for FTIR with diamond crystal) and using spectral range of $4000 \sim 400 \mathrm{~cm}^{-1}$ with resolution of $4 \mathrm{~cm}^{-1}$. The FTIR spectra of leaf extracts taken before and after synthesis of PdNPs were analyzed which was discussed for the possible function groups for the formation of S-PdNps, S-PdNp-LA, S-PdNp -LA-Vit

\section{SEM observations}

Samples of the aqueous suspension of palladium nanoparticles were prepared by placing a drop of the centrifuged suspension on carbon- coated copper grids and allowing water to evaporate. SEM observations were performed on a Zeiss EVO 40 Electron Microscope.

\section{Results and Discussion}

The biosynthetic method employing plant extracts have drawn attention as a simple and viable alternative to chemical procedures and physical methods. Such reliable and eco-friendly methods help in endorsing extra interest in the synthesis and application of nanoparticles which are good for mankind. Metal nanoparticles can be synthesized by reducing metal ion using some chemical molecules. In green synthesis, it is believed that natural material extract act as reducing agent for the generation of metal nanoparticles. In this work we have shown the ability of the solanum phytochemicals to interact with palladium chloride ions and reduce them to form palladium nanoparticles.

The reaction media offered a change in color from yellow (before the addition of palladium chloride, see Fig. 1a) to brown (after complete reduction of $\mathrm{PdCl}_{2}$ ions in the solanum leaf broth-Fig. 1b). The colour turned to dark brown when the S-PdNps was conjugated with lipoic acid (see Fig. 1c) and still darker with addition of vitamins to it (see Fig. 1d). The stability of palladium nanoparticles can be attributed to the formation of stable bonding between metallic palladium and reducing sugars, the phytochemicals such as saponins, tannins, terepenoids, flavonoids, anthocyanins, betacyanins, proteins and steroids and phenolic acids present in the leaf broth of Solanum trilobatum [30]. Sathish kumar et al., 2009 [31] hypothesized terpenoids to be the surface-active molecules that stabilized the palladium
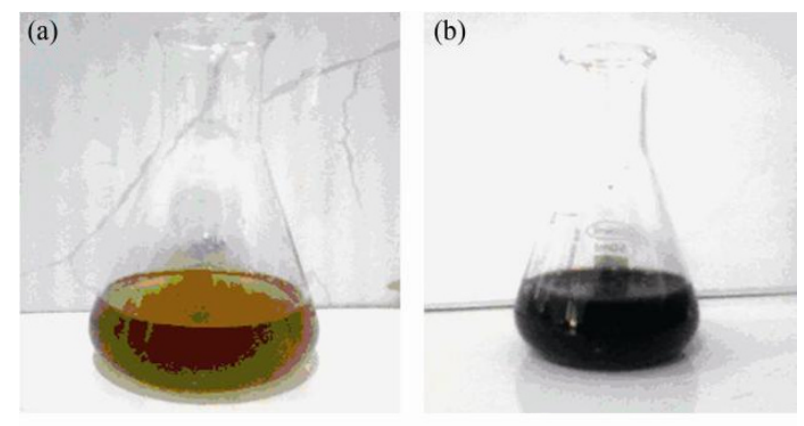

(c)

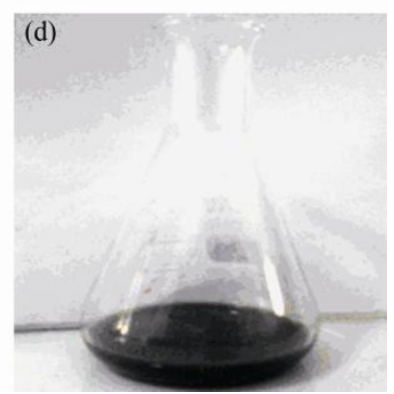

FIG. 1. Synthesis of PdNp from Solanum trilobatum leaf extract (a) Solanum trilobatum leaf extract before adding $\mathrm{PdCl}_{2}$ (Yellow); (b) Synthesis of PdNp from Solanum trilobatum leaf extract (Brown); (c) Solanum trilobatum leaf extract -Pd-Lipoic acid, (Dark brown); (d) Solanum trilobatum leaf extract -Pd-Lipoic acid-vitamin (Darker brown)

nanoparticles, and that the reduction of metal ions is possibly facilitated by these and/or sugars. Therefore, many plant extracts can be used to synthesize metal nanoparticles owing to the existence of phytochemicals and reducing sugars in them. 
We also made an attempt of conjugating palladium to lipoic acid, a thiol rich molecule which exhibited a dark brown colour than that of unconjugated palladium (see Fig. 1c). Chen et al., 1984 [32] in his study used a shorter chain thiol $\left(\mathrm{C}_{6} \mathrm{H}_{13} \mathrm{SH}\right)$ to protect the surface of palladium. As thiol cap (lipoic acid) enables weaker Pd-S bonding interactions it can easily break from the surface of the Pd nanoparticles during a longer reaction time. Studies have evidently illustrated that glutathiones (a thiol rich molecule) used for capping gold quantum clusters (AU-n-SG-m) (-SG, glutathione thiolate) has been well known for the stability of the AUNPs synthesized chemically. In addition the electrons present in the conduction band of nanoparticulate gold makes them potential candidates to bind with thiols and amines [33]. We have deduced from these observations that the aggregation of palladium nanoparticles and thiol-capped palldium nanoparticles in our present work could be due to the high $\mathrm{Pd}=\mathrm{C}_{18}: \mathrm{H}_{37}: \mathrm{SH}$ molar ratios resembling the formation of thiol-protected gold nanoparticles [34]. As a result, palladium nanoparticles may be easily tagged with various proteins and bio-molecules rich in amino acids leading to important biomedical applications. Formation of palladium nanoparticles and its complexes were centrifuged at $8000 \mathrm{rpm}$
Reduction of the aqueous palladium chloride ions was tracked by monitoring changes in color with UV-Vis spectroscopy. The relationship between the UV-visible radiation absorbance characteristics and the absorbent's size and shape is well established. Fig. 2a, 2b, and $2 c$ shows the UV-vis spectra recorded from the aqueous palladium chloride-Solanum leaf broth reaction medium as a function of time of reaction. After completion of the reaction, the wavelength of the surface plasmon band stabilizes at $270 \mathrm{~nm}, 240 \mathrm{~nm}$ and 200nm for S-PdNP, S-PdNP-lipoic acid and S-PdNP-lipoic acid-vitamin respectively. The nanoparticle absorption band is slightly asymmetrical with indications of an additional weaker component at $370 \mathrm{~nm}$. The presence of this shoulder indicates either formation of stable aggregates of the palladium nanoparticles in solution or shape anisotropy in the particles.

The intensity of the color arises from the surface plasmons, which are dipole oscillation arising when an electromagnetic field in the visible range is coupled to the collective oscillations of conduction electrons [35]. The broad surface plasmon peak is well known for the metal nanoparticles in size ranging from 2 to $100 \mathrm{~nm}$ and the absorption peak at $320 \mathrm{~nm}$ correspond to the wavelength at which the real and imaginary parts of the dielectric
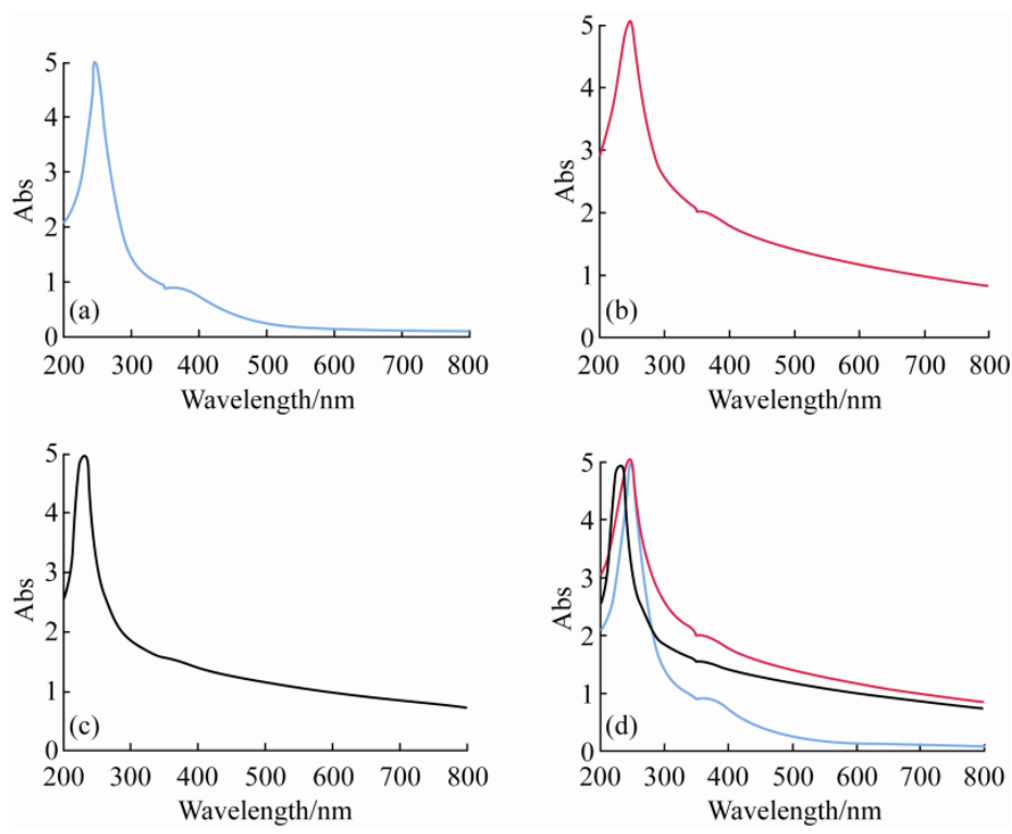

FIG. 2. UV-Visible Spectroscopy images of palladium nanoparticles from Solanum trilobatum leaf extract. (a) Synthesis of palladium nanoparticles from Solanum trilobatum leaf extract; (b) Conjugation of lipoic acid in Solanum trilobatum palladium nanoparticles; (c) Conjugation of vitamins along with lipoic acid in Solanum trilobatum palladium nanoparticles; (d) Combination of (a), (b) and (c).

for 10 min separately to isolate the palladium nanoparticles free from proteins or other compounds present in the solution. The pellets obtained after centrifugation were redispersed in water prior to further analysis. function of metals almost vanish [36]. With the increase in the particle size, the optical absorption spectra of metal nanoparticles that are dominated by surface plasmon resonances may shift towards longer wavelengths. Therefore the position of absorption band also strongly depends upon dielectric constant 
of the medium and surface-adsorbed species [37]. Based on Mie's theory, spherical nanoparticles give rise to a single surface plasmon resonance band in the absorption spectra, whereas anisotropic particles confer two or more surface plasmon resonance bands depending on the shape of the particles [38]. In present investigation, all reaction mixtures show a single surface plasmon resonance band revealing spherical shape of silver nanoparticles, which is addressed through SEM images.

The $\lambda$ max shift in the absorbance spectra observed in Fig. $2 \mathrm{~b}(240 \mathrm{~nm})$ and $2 \mathrm{c}(200 \mathrm{~nm})$ may possibly be due to the surface modification of the S-PdNps. The surface plasmon resonance being the major cause for the absorption is affected by surface modification with covalent coupling. The covalent coupling can be extended either via the carboxylic or the amino groups of lipoic acid/vitamins. In the case of lipoic acid capped nanoparticles, the disulfides are reduced by the palladium chloride ions to two thiol groups $(-\mathrm{S}-\mathrm{S}-\rightarrow-\mathrm{SH}+-\mathrm{SH})$, which may be involved in the binding of lipoic acid to S-PdNps. This type of capping can be of $\mathrm{pH}$ dependent and may lead to the precipitation of nanoparticles [33]. Thus the phenolics and other phytochemicals within Solanum trilobatum not only result in effective reduction of palladium ions to nanoparticles but their chemical framework is also effective at wrapping around the S-PdNps to provide excellent robustness against agglomeration. Therefore, by changing the size and shape of S-PdNps, the SPB and scattering may be tuned for application in cellular imaging, drug delivery and therapy. Figures $3 a, 3 b$, and $3 c$ indicate the size and morphology of S-PdNP, S-PdNP LA, and S-PdNP-LA-Vit respectively. SEM measurements on these particles expelled a spherical shape within the size range of $60 \sim 70 \mathrm{~nm}$ (see Fig. 4a).
65 80 nm (see Fig. 4b) and 75 100 nm (see Fig. 4c) which can be assigned to bioorganic compounds present in the leaf broth [32]. Such size distribution analysis of capped (PdNPs capped with lipoic acid and vitamins) and non-capped PdNPs confirms that the particles are well dispersed.

The analysis of IR spectra gives an idea about biomolecules bearing different functionalities which are present in underlying system. The FTIR spectra of samples containing PdNPs and its conjugates are illustrated in Fig. 4a-4c. Comparison between spectra of samples (S-PdNP, S-Pd NP-lipoic acid and S-Pd NP-lipoic acid-vitamin) reveals only minor changes in the positions as well as on the magnitude of the absorption bands. The FTIR spectrum of the S-PdNP showed bands at 661, 1584, $1637,2377,2675$ and $3473 \mathrm{~cm}^{-1}$ along with other small bands (see Fig. 4a). The intense band absorbance at $3473 \mathrm{~cm}^{-1}$ is the characteristic of the hydroxyl functional group in alcohols and phenolic compounds and the band at $1637 \mathrm{~cm}^{-1}$ can be assigned to the amide I band of the proteins released by the Solanum leaves or to $\mathrm{C}=\mathrm{C}$ groups/aromatic rings. In addition some weaker bands centered at 1584 and $2377 \mathrm{~cm}^{-1}$ characteristic to the amide III and amide I band in proteins also indicates a small concentration of protein in the PdNP solution synthesized using solanum leaf extract [39]. Effectively the C-N stretching vibration of aliphatic amines or to alcohols/phenols coincides with the band at $661 \mathrm{~cm}^{-1}$. Thus it is obvious that bands at 1637 and $2675 \mathrm{~cm}^{-1}$ assigning to the carbonyl groups and secondary amines, respectively signifies that PdNP synthesized using the Solanum leaf extract are surrounded by some proteins and metabolites having functional groups of amines, alcohols, ketones, aldehydes, and carboxylic acids.
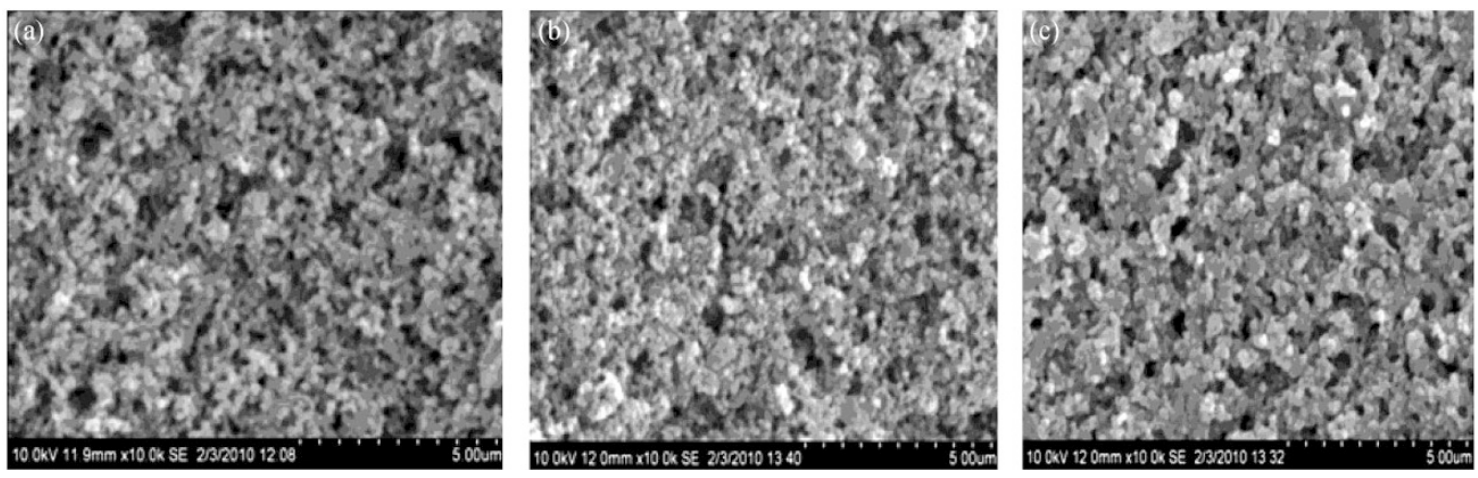

FIG. 3. SEM images of Palladium nanoparticles synthesised from Solanum trilobatum leaf extract at 10X magnification (a) Solanum trilobatum leaf extract-Palladium Chloride (60 70 nm); (b) Solanum trilobatum leaf extract-Palladium nanoparticles-Lipoic acid (65 80); (c) Solanum trilobatum leaf extract-Palladium nanoparticlesLipoic acid-Vitamin $(75 \sim 100 \mathrm{~nm})$. 


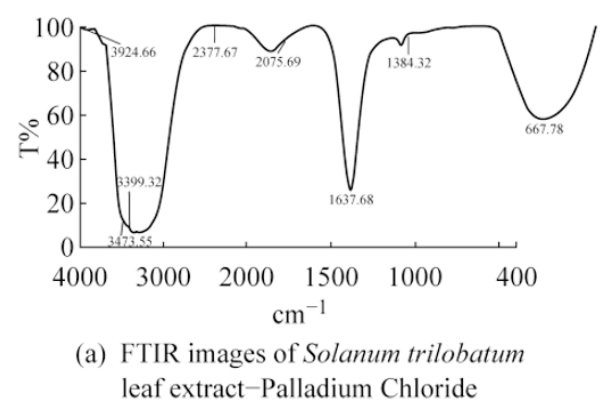

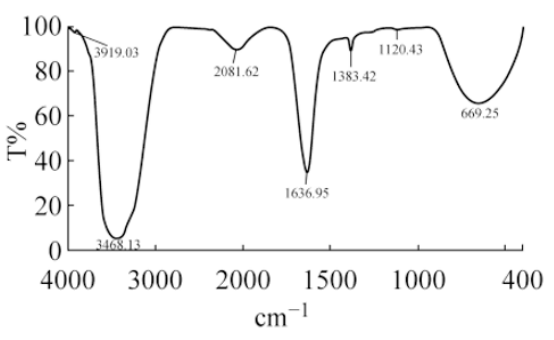

(b) FTIR images of Solanum trilobatum leaf extract-Palladium NanoparticleLipoic acid

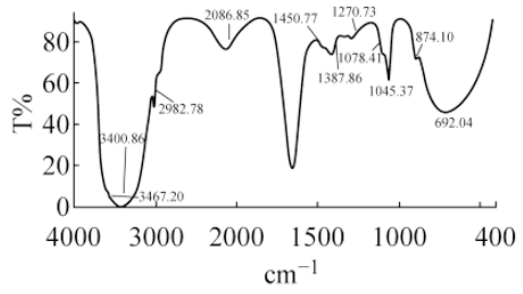

(c) FTIR images of Solanum trilobatum leaf extract-Palladium NanoparticleLipoic acid-Vitamin

FIG. 4. FTIR images of Palladium Nanoparticles Synthesised from Solanum trilobatum leaf extract.

On the other hand the spectrum of S-PdNP-lipoic acid explains the presence of six bands (see Fig. 4c) which clearly demonstrates the absence of the $-\mathrm{N}-\mathrm{H}$ stretch assingned to amide group (band at $3400.86 \mathrm{~cm}^{-1}$ ) and $\mathrm{S}=\mathrm{O}$ stretch of Sulfoxides (band at $1045.37 \mathrm{~cm}^{-1}$ ), but the presence of $\mathrm{CH}_{3}$ bend corresponding to Alkanes (band at $1383.95 \mathrm{~cm}^{-1}$ ) and the C-O stretch assigning to alcohols (band at $1120.43 \mathrm{~cm}^{-1}$ ) on comparing with the peaks of S-PdNP (see Fig. 3a). Deficient of $\mathrm{S}=\mathrm{O}$ stretch assigned to sulfoxides (band at $1045.78 \mathrm{~cm}^{-1}$ ) but the occurrence of $\mathrm{C}-\mathrm{O}$ stretch contributing to alcohols (band at $1107.73 \mathrm{~cm}^{-1}$ ) when compared with the peaks of S-PdNP (see Fig. 3a) were observed in the spectral bands of S-PdNP-Lipoic acid-vitamin (see Fig. 3c). The band around $1120.43 \mathrm{~cm}^{-1}$ and $1107.73 \mathrm{~cm}^{-1}$ in Fig. $3 \mathrm{~b}$ and Fig. $3 \mathrm{c}$ respectively can be assigned to $\mathrm{C}=\mathrm{O}$ stretching vibrations of the carbonyl functional group in ketones, aldehydes, and carboxylic acids [40,41,43]. The band around $1120.43 \mathrm{~cm}^{-1}$ and $1107.73 \mathrm{~cm}^{-1}$ matches to the ether linkages or -C-O- [42-43], whereas, the band around 1383.95 $\mathrm{cm}^{-1}$ and $1390 \mathrm{~cm}^{-1}$ corresponds to geminal methyls [42].

To a large extent, the band at $1069 \mathrm{~cm}^{-1}$ might be contributed by the -C-O-groups of the polyols such as flavones, terpenoids and the polysaccharides present in the biomass [43]. The absorbance band centered at $1636 \mathrm{~cm}^{-1}, 1639 \mathrm{~cm}^{-1}$ and $1638 \mathrm{~cm}^{-1}$ (3a, 3c and 3e) is associated with the stretching vibration of $-\mathrm{C}=\mathrm{C}$ - or aromatic groups [42-43]. These bands are due to amide I bond of proteins, indicating predominant surface capping species having $-\mathrm{C}=\mathrm{O}$ functionality which are mainly responsible for stabilization. A broad intense band around $3473.55 \mathrm{~cm}^{-1}, 3430.51 \mathrm{~cm}^{-1}, 3400.86 \mathrm{~cm}^{-1}$ and $3431.74 \mathrm{~cm}^{-1}$ in $3 \mathrm{a}, 3 \mathrm{~b}$ and $3 \mathrm{c}$ respectively can be assigned to the $\mathrm{N}-\mathrm{H}$ stretching frequency arising from the peptide linkages present in the proteins of the extract [44]. The shoulders around the band can be identified as the overtone of the amide-II band and the stretching frequency of the $\mathrm{O}-\mathrm{H}$ band, possibly arising from the carbohydrates and/or proteins present in the sample. However, although there is adequate evidence for the presence of surface bound proteins, the conclusion regarding the presence of the specific compound cannot be made unless the individual component is isolated and identified.

The spectras exhibiting broad asymmetric band around $2100 \mathrm{~cm}^{-1}$ can be assigned to the N-H stretching band in the free amino groups of PdNPs [44]. Since these band disappeared in the IR spectra of the final product it can be concluded that the reducing agents are largely derived from these water soluble heterocyclic compounds. Therefore, it reflects that water soluble heterocyclic compounds such as flavones are the reducing and capping ligands of the nanoparticles. Flavones could be adsorbed on the surface of the metal nanoparticles, possibly by interaction through $\pi$-electrons of carbonyl groups in the absence of other strong ligating agents in sufficient concentrations. Likewise, the oxygen atoms herein might facilitate the adsorption of the heterocyclic components on to the particle surface in stabilizing the nanoparticles [42]. Therefore it appears more likely that the reduction of palladium ions and stabilization of synthesized palladium nanoparticles is the responsibility of many functional groups, including amines, alcohols, ketones, aldehydes, alkenes and carboxylic acids, that are present in various plant metabolites and reducing sugars.

\section{Conclusion}

The rapid biological synthesis of palladium nanoparticles using leaf broth of Solanum trilobatum provides an economic and efficient route for the synthesis of nanoparticles with tunable optical properties. The approach of phytosynthesis appears to be cost efficient eco-friendly and easy alternative to conventional methods of palladium nanoparticles synthesis. The capping of bioorganic compound present in the leaf broth around each nanoparticle furnish uniform chemical environment for its stability. The palladium nanoparticles obtained were further characterized by conjugating with lipoic acid and vitamins. The nanoparticles thus synthesized from the herbal extracts can be used as an alternative to the chemically formulated palladium alpha-lipoic acid complex. However the beneficial effects of the 
herbally synthesized formulation against many age-related disorders (cardiovascular and neurodegenerative diseases) can be further explored using invitro and invivo studies.

Received 6 July 2010; accepted 30 August 2010; published online 19 September 2010.

\section{References}

1. D. Bhattacharya and R. K. Gupta, Crit. Rev. Biotechnol. 25, 199 (2005). doi:10.1080/07388550500361994

2. E. A. Rosei and M. L. Muiesan. Clin Exp Hypertens. 673, (2004). doi:10.1081/CEH-200031978

3. A. Leela and M.Vivekanandan, African J. Biotechnol. 7, 3162 (2008).

4. R. Narayanan and M. A. El-Sayed, J Phys Chem. B 10, 4357 (2005). doi:10.1021/jp044659t

5. V. Parashar, R. Parashar, B. Sharma and A. C. Pandey, Digest J. Nanomater. Biostruct. 4, 45 (2009).

6. P. Mohanpuria, N. Rana and K. S. K. Yadav, J Nanopart. Res.10, 507 (2008).

7. S. S. Shankar, A. Rai, A. Ahmad and M. Sastry, J. Colloid Interf. Sci. 275, 496 (2004). doi:10.1016/j.jcis. $\underline{2004.03 .003}$

8. J. L. Gardea-Torresdey, E. Gomez, J. Peralta-Videa, J. G. Parsons, H. E. Troiani and P. Santiago, Nano Lett. 2, 397 (2002). doi:10.1021/n1015673+

9. J. L. Gardea-Torresdey, E. Gomez, J. Peralta-Videa, J. G. Parsons, H. E. Troiani and P. Santiago, Langmuir, 19, 1357 (2003).

10. S. P. Chandran, M. Chaudhary, R. Pasricha, A. Ahmad and M. Sastry, Biotechnol. Prog. 22, 577 (2006). doi:10.1021/ bp0501423

11. B. Amkamwar, C. Damle, A. Ahmad and M. Sastry, J. Nanosci. Nanotechnol. 5, 1665 (2005). doi:10.1166/ inn.2005.184

12. S. Li, Y. Shen, A. Xie, X. Yu, L. Qiu, L. Zhang and Q. Zhang, Green Chem. 9, 852 (2007). doi:10.1039/ b615357g

13. S. S. Shankar, A. Rai, A. Ahmad and M. Sastry, Biotechnol. Prog. 19, 1627 (2003). doi:10.1021/ bp034070w

14. S. S. Shankar, A. Rai, A.Ahmad and M. Sastry, App. Nano Sci. 1, 69 (2004).

15. J. Y. Song and B. S. Kim, Korean J. Chem. Eng. 25, 808 (2008). doi:10.1007/s11814-008-0133-Z
16. J. Y. Song, H-K. Hyeon-Kyeong Jang and B. S. Kim, Process Biochem. 44, 1133 (2009). doi:10.1016/j.procbio. 2009.06.005

17. K. B. Narayanan and N. Sakthivel, Mater. Lett. 62,4588 (2008). doi:10.1016/j.matlet.2008.08.044

18. B. S. Kim, J.Y. CRC Press, Boca Raton, 399 (2009).

19. P. Mohanpuria, N. K. Rana and S. K. Yadav, J. Nanopart Res. 10, 507 (2008). doi:10.1007/s11051-007-9275-X

20. M. N. Nadagouda and R. S. Varma, Green Chem. 10, 859 (2008). doi:10.1039/b804703k

21. L. Jia, V. Wang and N. He, Nanotechnology 20, 385601 (2009). doi:10.1088/0957-4484/20/38/385601

22. N. Mallikarjuna, S. Nadagouda and Rajender, Varma npeng Wang, N. He and L. Ji, Green Chem. 10, 859 (2008). doi:10.1039/b804703k

23. M. Garnett, J. Inorg. Biochem. 59, 48 (1995). doi:10.1016/0162-0134(95)97337-P

24. G. wade, and M. Garnett, "Charge relay from molybdate oxiradicals from palladium-lipoic complex to DNA" paper presented on conference on Oxygen metabolites in matabollichemistry, univ. of minnesota, (1996).

25. M. Garnett, Garnett Mc. Keen Med. Sci. Ser. 1, 1 (2002a).

26. F. J. Antonawich and P. D. Valane, Anti-Aging Ther. 9, 13 (2007).

27. F. J. Antonawich, S. M. Fiore and L. M. Welicky, Exp. Neurol. 189, 10 (2004). doi:10.1016/j.expneurol. 2004.05.011

28. N. P. Sudheesh, T. A. Ajith, K. K. Janardhanan and C. V. Krishnan, Food and Chem. Toxicol. 47, 2124 (2009). doi:10.1016/j.fct.2009.05.032

29. N. P. Sudheesh, T. A. Ajith, K. K. Janardhanan and C. V. Krishnan, Food and Chem. Toxicol. 48, 1858-1862 (2010). doi:10.1016/j.fct.2010.04.022

30. B. Monavalli1, A. Raja Rajeswari, V. Gowri and A. Kanchana, J. Natural Sci. Technol. Life Sci. Bioinform. 2, 168 (2010).

31. M. Sathishkumar, K. Sneha, In Seob Kwak, Juan Mao, S. J. Tripathy and Y.-S. Yun, J. Hazardous Mater. 171, 400 (2009). doi:10.1016/j.jhazmat.2009.06.014

32. 1A. Chen, M. Goldbreg, N. Horwitz, A. Jawahery, P. Lipari, G. C. Moneti and H. Hecke, Phys. Rev. Lett. 52, 1084 (1984). doi:10.1103/PhysRevLett.52.1084

33. Mee-Kyung Chung and M. Schlaf, J. Am. Chem. Soc. 126, 7386 (2004). doi:10.1021/ja049386u 
34. M. Abubaker, H. Muhammed, S. Ramesh, S. S. Sinha, S. K. Pal and T. Pradeep, Nano Res 1, 333 (2008). doi:10.1007/s12274-008-8035-2

35. P. Mulvaney, Langmuir. 12, 788 (1996). doi:10.1021/ $\underline{\text { la9502711 }}$

36. S. C. Prathap, M. Chaudhary, R. Pasricha, A. Ahmad and M. Sastry, Biotechnol. Prog. 22, 577 (2006). doi:10.1021/ bp0501423

37. Y. Xia and N. J. Halas, Mrs. Bull. 30, 338 (2005).

38. G. Mie and A. D. Physik. 25, 377 (1908). doi:10.1002/ andp.19083300302

39. M. van de Weert, P. I. Haris, W. E. Hennink and D. J. A. Crommelin, Anal. Biochem. 297, 160 (2001). doi:10.1006/abio.2001.5337
40. S. Shiv Shankar, A. Ahmad and M. Sastry, Biotechnol. Prog. 19, 1627 (2003). doi:10.1021/bp034070w

41. S. Shiv Shankar, A. Rai, A. Ahmad and M. Sastry, J. Colloid Interf. Sci. 275, 496 (2004). doi:10.1016/ j.jcis.2004.03.003

42. J. Huang, Q. Li, D. Sun, Y. Lu, Y. Su, X. Yang, H. Wang, Y. Wang, W. Shao, N. He, J. Hong and C. Chen, Nanotech. 18, 1 (2007). doi:10.1088/0957-4484/18/40/405302

43. H. Yin, S. Zhang, J. Wu, H. Nan, L. Long, J. Yang and Q. Li, Molecules 11, 786 (2006). doi:10.3390/11100786

44. 4P. Mukherjee, M. Roy, B. P. Mandal, G. K. Dey, P. K. Mukherjee, J. Ghatak, A. K. Tyagi and S. P. Kale, Nanotechnology 19, 075103 (2008). 